\title{
Concept Recognition with Convolutional Neural Networks to Optimize Keyphrase Extraction
}

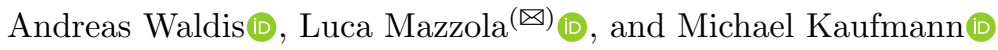 \\ School of Information Technology, \\ Lucerne University of Applied Sciences, 6343 Rotkreuz, Switzerland \\ \{andreas.waldis, luca.mazzola, m.kaufmann\}@hslu.ch
}

\begin{abstract}
For knowledge management purposes, it would be useful to automatically classify and tag documents based on their content. Keyphrase extraction is one way of achieving this automatically by using statistical or semantic methods. Whereas corpus-index-based keyphrase extraction can extract relevant concepts for documents, the inverse document index grows exponentially with the number of words that candidate concepts can have. Document-based heuristics can solve this issue, but often result in keyphrases that are not concepts. To increase concept precision, or the percentage of extracted keyphrases that represent actual concepts, we contribute a method to filter keyphrases based on a pre-trained convolutional neural network (CNN). We tested CNNs containing vertical and horizontal filters to decide whether an n-gram (i.e, a consecutive sequence of $\mathrm{N}$ words) is a concept or not, from a training set with labeled examples. The classification training signal is derived from the Wikipedia corpus, assuming that an n-gram certainly represents a concept if a corresponding Wikipedia page title exists. The CNN input feature is the vector representation of each word, derived from a word embedding model; the output is the probability of an n-gram to represent a concept. Multiple configurations for vertical and horizontal filters are analyzed and optimised through a hyper-parameterization process. The results demonstrated concept precision for extracted keywords of between 60 and $80 \%$ on average. Consequently, by applying a CNN-based concept recognition filter, the concept precision of keyphrase extraction was significantly improved. For an optimal parameter configuration with an average of five extracted keyphrases per document, the concept precision could be increased from 0.65 to 0.8 , meaning that on average, at least four out of five keyphrases extracted by our algorithm were actual concepts verified by Wikipedia titles.
\end{abstract}

Keywords: Natural language processing - Concept recognition • Convolutional neural networks .

Keyphrase extraction Keyword extraction

\section{Introduction}

With the diffusion of user-generated content (UGC), the amount of information a knowledge management (KM) system should treat grows exponentially. 
On top of this, as the KM tool has no control over the source and format of the documents, it should be able to extract the major themes covered relying on some conceptualization process. To this end, the primary existing approach is called automatic document tagging and relies on the association amongst a series of keywords to each record based on their frequency and peculiarity for the document itself [28].

All-the-same, it is sometimes not advisable to rely on simple keywords, as they are unable to fully capture the semantic meaning of the subjects covered by an entry [4]. For this reason, we rely on the notion of concept, as a consecutive ordered sequence of single words. This is also known as n-gram, and the process for its identification as n-gram keyphrases extraction [26]. A concept is an idiomatic construction that conveys a meaning for humans used in this context for allowing them to have a feeling about the underlying subject(s). Building a comprehensive index of all the combinations of word stems for N-grams is a computationally complex problem because it grows over-linearly (combinatorially) with the increase of the length (n) of the sequence. Therefore, it is not feasible to tackle the problem in this way for a real case.

In this work, we measure the effects of applying a Convolutional Neural Network (CNN) as a filter on the set of the N-grams computed by a heuristic document based keyphrase extraction method with the objective to reduce the rate of false positives, that is, the percentage of keyphrases that are not concepts. The idea is to rely on the well-known capabilities of CNN to extract patterns from images [27] to use the set of extracted characteristics for identifying complex patterns in the construction of concepts as valid N-grams combination. A similar approach is already known in literature [22], where the objective is to identify matching in written text, treated as images.

The purpose of this research is to evaluate a neural network based algorithm to decide whether consecutive sequences of $\mathrm{N}$ words represent concepts. In this context, a concept is an idiomatic construction that conveys a meaning for humans. We use the English Wikipedia as labeled training corpus, We assume that all Wikipedia entry titles are concepts and our algorithm uses the existence of a Wikipedia entry for a given word combination as training signal. The resulting neural network should be able to recognize N-gram concepts in a given text. Those concepts can be used for entity extraction and automatic tagging without building a huge N-gram-based inverse document index. Such an algorithm that delivers proper n-gram concepts, regardless of the category and the size of the corpus, can increase the usefulness of keyphrases extraction for (semi-)automatic document classification or summarisation. In this work, we build upon a previously published conference paper [30], where the approach for concept recognition with convolutional neural networks has been described in detail. In the following sections describe our approach for keyphrase extraction and the application of CNN concept recognition as well as the measured effects based on data collected from experiments applying this new approach to a collection of Wikipedia articles. In Sect. 5 we summarize our insights from this research. 
The rest of this paper is organised as follows: Sect. 2 explores the state of the art, in particular introducing the subjects of keyphrase extraction, automatic concept recognition, word embeddings, and the basics of convolutional neural networks. Most part of this section has been published by [30]. Section 3 is devoted to present concept recognition with CNN, from introducing our architecture, till evaluating its performance in isolation. Again, this Section is an excerpt from our conference paper [30], to make the basics clear for the reader. The new keyphrase extraction method with and without CNN is covered in Sect.4, also using a toy demonstrative example. We present an experimental part by examining the performance impact of $\mathrm{CNN}$-based filtering for keyphrase extraction based on collected data. This Section provides the core of our original contribution for this chapter. Some lessons learned and future steps that we would like to take conclude this paper in Sect. 5 .

\section{State of the Art}

\subsection{Keyphrase Extraction}

Keyword extraction is the task of evaluating which words summarize the aboutness, or topics, of a text. However, often topics consist of more than one words, or n-grams. Keyphrase extraction aims at evaluating the central aspects of a text by ranking word groups regarding their relevance. Several approaches exist. Clustering-based methods such as Liu et al. [17] automatically group documents together and give clusters a label. Linguistic approaches such as [10] use grammatical structures based on part-of-speech tagging to generate keyphrases, and apply metrics based on statistical distribution to evaluate the relevance of keyphrases for the text. Graph-based methods [1] model documents as a graph, where nodes are words or phrases and edges are some form of relation between nodes, such as co-occurrences. For example, Text Rank [19] uses single words as nodes and co-occurrences in a window of two words as relationships. The importance of keyword candidates is then evaluated by a score based on the ratio between the sum of incoming edges weight divided by the sum of outgoing edges weight. In a post-processing phase, keyphrases are generated by joining direct sequences of $\mathrm{n}$ keywords in the text, to form n-grams. Corpus-based rankings such as TF-IDF evaluate the relevance of keywords and keyphrases based on the distribution of the symbols in the corpus [15]. On the other hand, documentbases approaches such as RAKE (Rapid Automatic Keyword Extraction) [25] operate only on distributions of candidate keywords in the document. RAKE computes more efficiently and achieves higher precision. The RAKE algorithm evaluates the relevance of keywords by dividing the frequency of a keyword candidate by the frequency of its co-occurrence with other keyword candidates. In a post-processing phase, keyphrases are extracted by joining keywords that occur at least twice in the same sequence, including the stop words between them. Keyphrase extraction methods usually focus on ranking keywords for relevance, and only apply simple heuristics or postprocessing to combine single words to keyphrases. Using concept recognition techniques, it is possible to generate more 
sophisticated methods to decide whether word combinations are concepts, which can improve keyphrase extraction.

\subsection{Automated Concept Recognition}

The importance of n-grams for text classification was shown already 20 years ago [7]. Many statistical and semantic methods have been proposed for concepts extraction. The use case described by [32] is an example for the usage of traditional neural networks, and [5] for the statistical approach. More recently, deep learning (an extension of neural networks with multiples hidden layers) is gaining relevance for all aspects of NLP, as mentioned by [18]. Concept recognition, as sub-field of concept mining, divides phrases into sequences of consecutive words classified as concepts and non-concepts. According to [23] concepts are useful by providing standalone information, in contrast to any random non-concepts. This information, as in [4], can be categorized as object, entity, event, or topic. For instance, the string "the Guardian newspaper was founded in 1821" contains 28 n-grams with the length of one to seven. The concept "Guardian newspaper" is one of them and has a significantly higher information level than the nonconcept "newspaper was founded in". There are several different approaches for deciding whether a phrase is a concept. [23] showed a combination of linguistic rules and statistical methods. The authors defined these rules to characterize possible concepts and filter out non-concepts. For example, a candidate has to contain a minimum of one noun and is not allowed to start or end with a verb, a conjunction, or a pronoun. After filtering out non-candidates, the remaining ones are judged by their relative confidence. This is a metric to help deciding if a sub/super-concept of the candidate actually fits better as a concept. For example, "Guardian newspaper" is a better choice than "Guardian newspaper was founded in 1821" because of the higher relative confidence. Another method is shown in [16] with regards to Chinese bi-grams. Like [23] they combine statistical methods with linguistic rules as well, but in contrast, they first calculate the statistical metric and then filter out the results with linguistic rules. For measurement, they used the mutual information $(M I)$ and document frequency $(D F)$ metrics. $M I$ represents the joint probability with respect to the product of the individual probabilities, for two words in a bi-gram. Since $M I$ tends to prefer rare words, they used the $D F$ value to reduce the influence of low-frequency words, as it takes into account the number of documents containing a bi-gram, normalized by the total number of documents.

\subsection{Word Embeddings}

Embedding $f: X \hookrightarrow Y$ map an object from a space $X$ to another object of the space $Y$. One of the usages of embeddings in the field of NLP is, for example, to map a word (an item of the space of all words) to a vector in a high-dimensional space. Since these vectors have numerical nature, a wide range of algorithms can use them. The three mainly used embedding algorithms are Word2Vec [24], GloVe [31], and fastText [12]. While GloVe uses statistical information of a word, 
Word2Vec and fastText adopt co-occurrence information to build a model. They calculate word embeddings based on either the continuous bag of words $(\mathrm{CBOW})$ model or the skip-gram model of [20]. Those latter models predict respectively a word based on surrounding words $(C B O W)$ or the surrounding words based on one word (skip-gram). CBOW and skip-gram rely on an input matrix $\left(W_{I}\right)$ and an output matrix $\left(W_{O}\right)$ as weight matrices. Those randomly initialized matrices are updated after each training iteration. The purpose of these matrices is to connect the neural network input layer to the hidden layer through $W_{I}$ and the hidden layer to the output layer through $W_{O}$. In both methods $W_{I}$ has the dimensions $V \times N$ and $W_{O}$ the dimensions $N \times V$, where $V$ represents the size of the vocabulary and $N$ the size of the hidden layer. After optimizing these weight matrices, they can be used as a dictionary to obtain a vector for a specific word $\boldsymbol{h}=\boldsymbol{x} * W_{I}$, as discussed in [24].

Nevertheless, Word2Vec is only able to compute vectors for trained words, as it uses the vector of the whole word. One main advantage of fastText is the possibility of getting a word vector of an unknown word. To achieve this, it uses the vector's sum of sequences of included characters of one word, instead of one word as a whole. For example, where, enriched by the padding symbols $<$ and $>$, is represented by $<w h$, whe, her, ere, and er $>$.

\subsection{Convolutional Neural Networks}

As a variation of neural networks (NNs), convolution neural networks (CNNs) are often used in computer vision for tasks such as image classification and object recognition. Usually, they adopt a matrix representation of an image as an input and a combination of different hidden layers to transform the input into a certain category or object. These layers are used to analyze specific aspects of the image or to reduce its dimensionality. Word embedding enables the numeric representation of words as vector, and the representation of n-grams as a matrix. Furthermore, they can serve as an input for CNNs. $[9,13,14]$ have shown various use cases for the usage of CNNs in language modeling and text classification. They all rely on the word vectors as a matrix input. Inside the network, they combine different layers to analyze the input data and reduce the dimensionality. Two main processes are used in the network to learn: Forward propagation represents the calculation process throughout the whole $\mathrm{NN}$ to predict the output data for given input data. Each layer of the network takes the output of the previous layer and produces its updated output. The next layer uses this output as a new input. This process continues until it reaches the last layer. A majority of the layers use a weight matrix to process this transformation. This weight matrix controls the connection; that is, the strength between the input neurons and the output neurons. Finally, the update over the time of these weight matrices represents the learning process of the entire network. Back propagation allows the adaption of the neuron's connections weight based on the error between the output label and the resulting prediction. The metric mean squared error is shown in Eq. 1, with $y_{p}$ as the predicted value and the truth as $t_{p}$ : 


$$
E=\frac{1}{n} \sum_{p}\left(y_{p}-t_{p}\right)^{2}
$$

The goal of the back propagation process is to adjust the network's weights to minimize the difference $y_{p}-t_{p}$. This is done by propagating the error value layer by layer back through the network, calculating the partial derivative of the path from the output to every weight. Equation 2 shows how the error of a network can be back propagated to the weight $w_{b_{3}, c}$ :

$$
\frac{\partial E}{\partial w_{b_{3}, c}}=\frac{\partial E}{\partial c_{o u t}} * \frac{\partial c_{o u t}}{\partial c_{\text {in }}} * \frac{\partial c_{\text {in }}}{\partial w_{b_{3}, c}}
$$

After distributing the output error over all weights, the actual learning takes place. Equation 3 shows that the new weight is the difference between the actual weight and the error multiplied by the learning rate:

$$
w_{b_{3}, c}=w_{b_{3}, c}-\left(l r * \frac{\partial E}{\partial w_{b_{3}, c}}\right)
$$

The kind of transformation and the connections inside the network are defined by the different kinds of layers used. The following layers are the mostly used ones: Convolution layers are used to analyze parts of or reduce the dimensionality of the input by applying a linear filter to the input matrix. This is done by iterating a kernel matrix $(K)$ of the dimension $k_{1} * k_{2}$ through the whole input matrix $(I)$. The kernel matrix represents the weights and is updated through backward propagation. After applying the convolution operation to the input matrix, the bias value adds the possibility of moving the curve of the activation function in the $\mathrm{x}$-direction and improve the prediction of the input data. Subsequently, the non-linear function adds some non-linearity. Without that, the output would be a linear combination of the input, and the network could only be as powerful as the linear regression. Doing that allows the network to learn functions with higher complexity than linear ones [21]. Pooling layers reduce the complexity and computation demands of the network, without involving any learning. The layer uses the given input matrix and creates a more compact representation of the matrix by summarizing it. It typically works with a $2 * 2$ window matrix iterating over the input matrix without overlapping. There are different kinds of pooling layers such as max-pooling or average-pooling. Using max-pooling, the highest value of the four cells serves as the representation of those cells instead of an average value. Dropout layers randomly ignore a percentage of the input neurons. This process only happens during the network training, for validation and prediction. As [29] showed, applying the dropout mechanism in a network increases the training duration but also increases the generality and prevents overfitting the network to the training set. The dropout procedure changes for each training sequence, as it is dependent on the input data. Flatten layers reduce the dimensionality of the input. For example, they convert a tri-dimensional input $(12 \times 4 \times 3)$ into a bi-dimensional ones $(1 \times 144)$. Dense layers are used to change the size of the given input vector. This dimensionality change is produced 
by connecting each row of the input vector to an element of the output vector. This linear transformation uses a weight matrix to control the strength of the connection between one input neuron and the output neurons. Like convolution layers, a bias value is added after the transformation, and an activation function adds some non-linearity. Dense layers are often used as the last layer of the network to reduce the dimensionality to usable dimension to get the prediction value, for example, with the softmax activation function, mostly used to get the predicted category in a classification task.

\section{Concept Recognition with Convolutional Neural Networks}

N-gram keyphrase extraction is a dimensionally hard problem. With increasing $\mathrm{n}$, more and more of the extracted n-grams tend to not represent concepts, which makes the corresponding keyphrases noisy. Training a neural network to recognize whether an n-gram is a concept or noise could help filter the space of candidate n-grams for keyphrase ranking. To achieve this, the following method was implemented to filter out keyphrases using a trained neural network so that more keyphrases actually represent concepts. The input of the neural network is represented by a list of all n-grams extracted from the English Wikipedia corpus, with a maximal length of 7 , encoded into a fixed 300-dimensional matrix by the word embedding model. The neural network is trained by using the set of Wikipedia page titles as the gold standard for deciding whether a sequence of words represents a concept: if an n-gram corresponds to a Wikipedia entry title, the training signal to the neural network is 1 ; else 0 . The output of the neural network, for each n-gram, is a prediction of whether it represents a concept or not, together with the probability. The goal is to maximize the precision of the concept list, to obtain a high hit rate. The objective is to support automatic document tagging with n-gram concept extraction. Evaluation of the neural network's output success, again, uses Wikipedia page titles. If the neural network classifies a word sequence as a concept, then this is a true positive (TP) if there is a Wikipedia page with this title; otherwise, it is a false positive (FP). If the network classifies an n-gram as a non-concept, then this is a true negative (TN) if there is no Wikipedia entry with that name, or else it is a false negative (FN).

\subsection{Neural Network Architecture}

Figure 1 shows the NN network architecture. The input matrix is fixed to the dimensions $7 \times 300$ and contains the vector representation of the $n$-grams. Since the network needs a fixed input size, whenever $n<7$, the matrix will be filled up with zero vectors. After specifying the input of the network, the convolution layers start analyzing the given data. For this purpose, two separate network paths are built for analyzing the data in the horizontal and vertical directions. Each of those two paths includes multiple convolution layers with different dimensions to gather different perspective of the data. All layers use a one-dimensional convolution layer that maps the two-dimensional inputs to a one-dimensional output. 


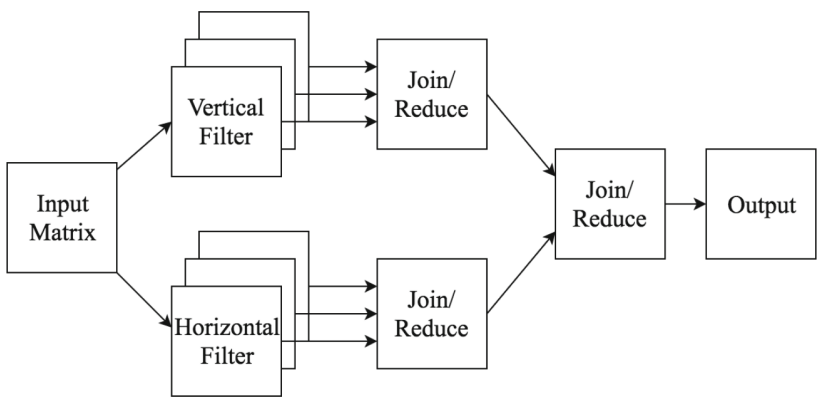

Fig. 1. Basic network structure (reprinted from [30]).

As shown in Fig. 2 the vertical convolution layers use filters with a fixed width of 300 and a dynamic height (here $2,3,4$ ). Correspondingly, the horizontal convolution layers are using a fixed height of 7 and a dynamic width (with typical values $30,50,70)$. The rectified linear unit ( $\operatorname{ReLU}), f(x)=\max (0, x)$, serves as
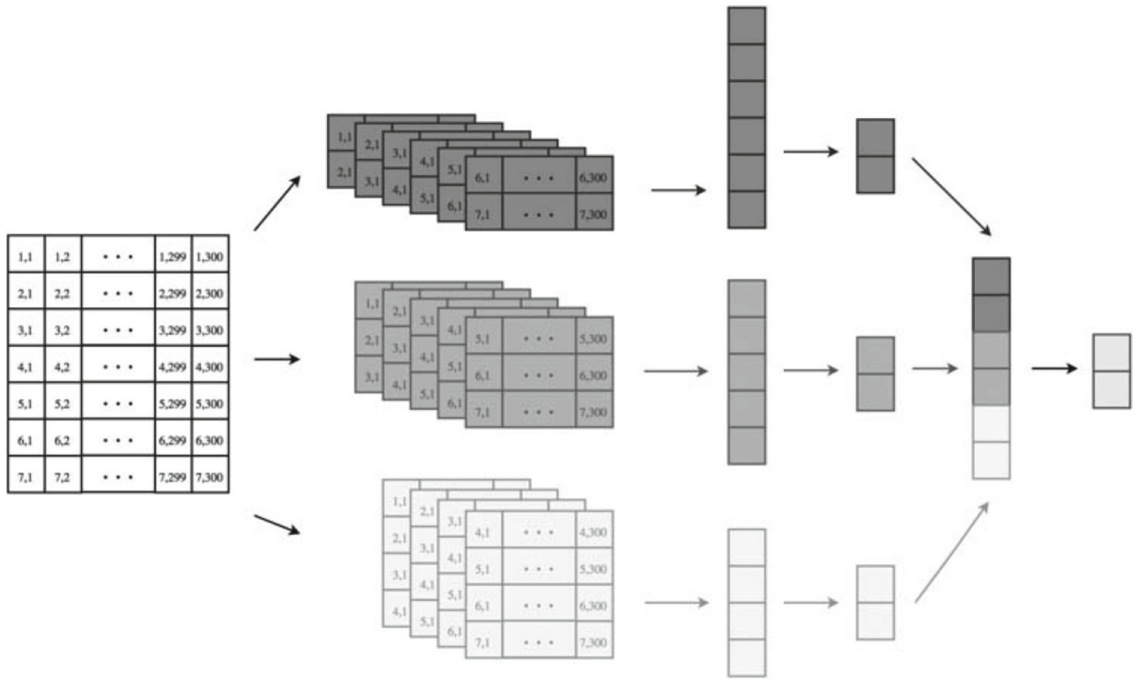

Fig. 2. Vertical convolution layers, with the different filter sizes (reprinted from [30]).

activation function for all filters, to add non-linearity to the output. Since the output dimension of the convolution layers is related to the filter size, the outputs of the filters are not balanced. For example, a vertical filter of size $2 \times 300$ produces an output vector of size 6 while an output vector for a filter size of $4 \times 300$ has length of 4 . To deal with this aspect, after the pooling layers have reduced the complexity, some dense layers decrease the length of the output vector of each horizontal and vertical filter to 2 , regularising them. They also use 
the ReLU function as their activation function. After reducing the dimensions, a merge layer joins all vectors of the horizontal and vertical paths. Then, the dimensionality of both resulting vectors will be reduced again to 2 by the usage of a dense layer. This eventually results in two vectors of length 2,1 for the horizontal path and 1 for the vertical path. To get the final prediction for the input, a join layer merges both vectors of the two paths into one vector with length 4 . Subsequently, a final output layer uses the softmax function as a dense layer (as seen in Eq. 4) to reduce the dimensions to 2. It squashes all values of the result vector between 0 and 1 in a way that the sum of these elements equals 1 . The processed result represents the probability of one input n-gram being marked as concept.

$$
\frac{\exp \left(a_{k}(\bar{x})\right)}{\sum_{j} \exp \left(a_{j}(\bar{x})\right)}
$$

\subsection{Word Embedding as N-Gram Features}

For NLP applications, the choice of a word embedding plays a fundamental role, while also holding some contextual information about the surrounding words. This information could enable an NN to recognize n-grams that it has never seen in this sequence, thanks to a previously seen similar combination. The following two models generate the vector representation of a word: Word2Vec is a pretrained 300-dimensional model without additional information hosted by [8]. Word2Vec-plus is an extended version of the pre-trained model from [8]. It uses words with a minimum frequency of 50 , extracted from a data set with 5.5 million Wikipedia articles. To get a vector representation $\boldsymbol{v}_{\boldsymbol{u}}$ for an unknown word, the approach uses the average vector representation of the surrounding four words, if they have a valid vector, or the zero vector, if they are also unknown (shown in Eq. 5).

$$
\boldsymbol{v}_{\boldsymbol{u}}=\operatorname{avg}\left(\sum_{\substack{i=-2 \\ i \neq 0}}^{2} \boldsymbol{v}_{\boldsymbol{i}}\right) \begin{cases}\boldsymbol{v}_{\boldsymbol{i}}, & w_{i}=\text { known word } \\ \boldsymbol{v}_{\boldsymbol{i}}=\mathbf{0}, & w_{i}=\text { unknown word }\end{cases}
$$

After averaging the unknown vector for one occurrence, the overall average $\boldsymbol{v}_{\boldsymbol{n}}$ will be recalculated. As shown in Eq. 6, the existing average $\boldsymbol{v}_{n-1}$ will be multiplied by the previous occurrences $w_{n-1}$ of the word and added to the vector calculated in Eq. 5. This value will be divided by the number of previous occurrences plus 1 , to get a updated overall average for the unknown word. This variant of the average calculation prevents large memory consumption for an expanding collections of vectors.

$$
\boldsymbol{v}_{n}=\frac{\boldsymbol{v}_{n-1} * w_{n-1}+\boldsymbol{v}_{\boldsymbol{u}}}{w_{n-1}+1}
$$

\subsection{Training and Experimental Setup}

Different network configurations were tested to find the best model for classifying concepts and non-concepts in our test case, according to the conceptual model described in the previous section. Figure 3 shows the iterative training pipeline: 


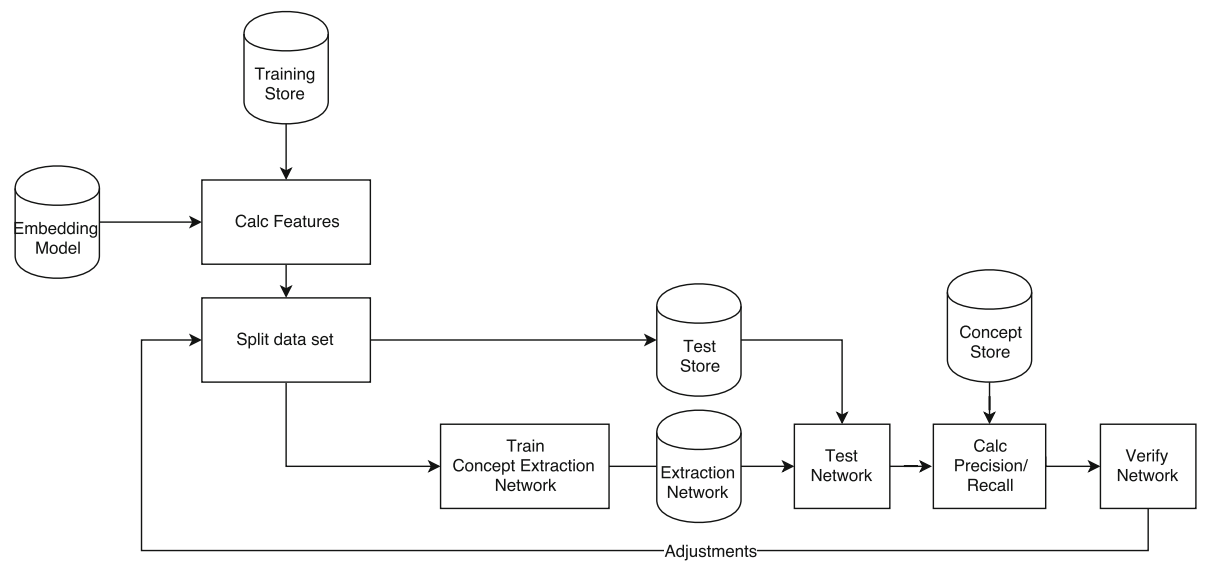

Fig. 3. The adopted training pipeline (reprinted from [30]).

1. Initially, the features of all n-grams found in the English Wikipedia corpus were calculated by extracting the corresponding word vectors from the embedding model.

2. Afterwards, the data set was separated into the training set (80\%) and the test set $(20 \%)$.

3. The training process used the training set to generate the extraction network. In this phase, the network was trained to recognize n-grams that are likely to represent a Wikipedia page title, based on their structure.

4. The prediction of the resulting network was based on the test set. During this process, all items of the test set were classified either as concepts or non-concepts.

5. The verification of the network was based on precision, recall, and $f 1$ metrics calculated during the previous evaluation.

6. To limit the human effort in classifying the results, we relied on the assumptions that valid concepts are statistically present as page names (titles) into Wikipedia, and that non-concepts are likely to not appear as page titles in this source.

7. Based on the performance comparison of a run with the previous ones, either the pipeline considered as finished or another round was started with an updated network structure.

The encoding used for presenting the data to the networks influences their performance, especially its ability for generalization. A good generalization depends mainly on the following three aspects:

- Data balancing: the input data is well-balanced by containing a similar amount of concept and non-concept examples. The final data set contains one million concepts and one million selected non-concepts. These two million samples do not fit completely into memory; thus dividing them into 40 
parts avoids a memory overflow. The training process loads all of these parts, one by one for each epoch.

- Data separating: existing samples are separated into the training part (80\%) and the test part $(20 \%)$ to prevent overfitting of the networks. Crossvalidation gives information about the level of generalization and mean performance. First of all, it separates the training part into four parts $(25 \%$ of the original $80 \%$ set). Each of them is used once as validation part, while the remaining three parts serve as training data for the network. The precision, recall, and f1 score give a weight to each run of the cross-validation process of each network. Further changes to the network structure are based on these values to improve the performance. Also, those metrics are used to select the better performing and most stable networks. A final training run on these network uses the whole training part as input data and the test part to produce a final measurement of the best networks.

- Shuffling of the input data helps to get early convergence and to achieve better generalization, as also mentioned by [2]. For this purpose, the training environment loads all training parts in each epoch in a new random order. Furthermore, it shuffles all examples inside each part before generating the batches to send as network inputs.

Table 1 specifies the different network configurations adopted to investigate how vertical (v-filters) and horizontal (h-filters) filters can affect the performance of the resulting network. The intervals of the parameters shown in Table 2 are considered during the training. The actual combination differs by use case or experiment and is based on well-performing sets experienced during the whole project.

Table 1. Different model combinations (Source [30]).

\begin{tabular}{l|l|l}
\hline Name & v-filters & h-filters \\
\hline V3H0 & $(2,3,4)$ & () \\
\hline V6H0 & $(2,3,4,5,6,7)$ & () \\
\hline V0H3 & () & $(100,200,300)$ \\
\hline V3H1 & $(2,3,4)$ & $(1)$ \\
\hline V3H3 & $(2,3,4)$ & $(100,200,300)$ \\
\hline V6H1 & $(2,3,4,5,6,7)$ & $(1)$ \\
\hline V6H3 & $(2,3,4,5,6,7)$ & $(100,200,300)$ \\
\hline V6H6 & $(2,3,4,5,6,7)$ & $(10,20,30,40,50,60)$ \\
\hline
\end{tabular}


Table 2. Hyperparametrisation (Source [30]).

\begin{tabular}{l|l}
\hline Parameter & Value \\
\hline Dropout & $0.1-0.5$ \\
\hline Learning Rate & $0.0001,0.0005$ \\
\hline Epochs & $100-400$ \\
\hline Batch Size & $32,64,128,256$ \\
\hline
\end{tabular}

\subsection{Evaluation of CNN Performance}

Figure 4 shows the resulting precision, recall, and $f 1$ values in the training phase for all vertical and horizontal filter combinations, after the 400 training epochs. Here, some differences emerge:

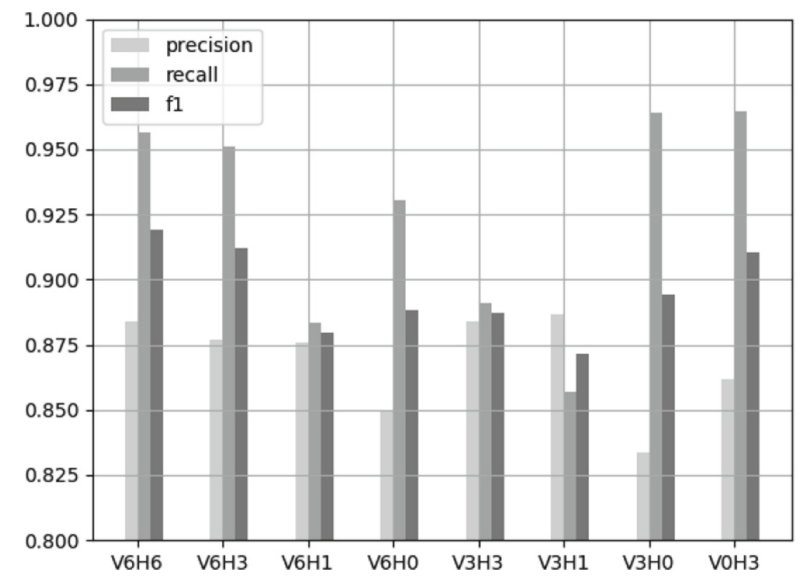

Fig. 4. Overall performance of the tested vertical and horizontal filters combinations in the training phase (reprinted from [30]).

- Based on precision, the V3H1 network had slightly better performance than V6H6 and V3H3, with a score of 0.8875 .

- Considering the recall, on the other hand, the V0H3 (0.9625) architecture outperformed V6H3 and V6H6.

- Using the $f 1$ score, V0H3, V6H3, and V6H6 networks outperformed all others. However, among them, none has a significantly better performance, with all in the range from 0.91 to 0.9175 .

Table 3 lists some classification examples, separated by their membership in the confusion matrix. True positive (TP) and true negative (TN) contain meaningful examples. The phrase "carry out" is an example of a concept that does 
Table 3. Examples of neural network output, divided into true positives (TP), false positives (FP), false negatives (FN), and true negatives (TN) with regards to existing Wikipedia page titles. Objective is to minimise the FP set (Source [30]).

\begin{tabular}{|c|c|c|}
\hline & Concepts & Non-Concepts \\
\hline & \begin{tabular}{||l} 
American Educational Research Journal \\
Tianjin Medical University \\
carry out \\
TP Bono and The Edge \\
Sons of the San Joaquin \\
Glastonbury Lake Village \\
Earl of Darnley
\end{tabular} & $\begin{array}{l}\text { to the start of World War II } \\
\text { must complete their } \\
\text { just a small part } \\
\text { FP a citizen of Afghanistan who } \\
\text { itself include } \\
\text { NFL and the } \\
\text { a Sky }\end{array}$ \\
\hline 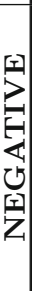 & $\begin{array}{l}\text { therefore it is } \\
\text { use by } \\
\text { in conversation with } \\
\text { Council of the Isles of Scilly } \\
\text { Xiahou Dun } \\
\text { The Tenant of Wildfell Hall }\end{array}$ & $\begin{array}{l}\text { Regiment Hussars } \\
\text { University of Theoretical Science } \\
\text { Inland Aircraft Fuel Depot } \\
\text { NHL and } \\
\text { MN } \\
\text { Mnican State Senate } \\
\text { University of } \\
\text { Ireland Station } \\
\text { In process }\end{array}$ \\
\hline
\end{tabular}

not make sense out of context, but there is a Wikipedia page about it. Similar phrases can be found from among the FP examples, such as "University of Theoretical Science" and "Mexican State Senate": they look like proper concepts but there is no Wikipedia entry with that title. They were probably selected because of the similar structure to some concepts. This also happened in the opposite direction; for example the phrase "in conversation with" is classified as non-concept based on the similarity with actual non-concepts; yet there is a TV series on $\mathrm{BBC}$ with the same name.

The test data set was restricted labeled data set, evaluating 20,000 selected n-grams for each of the networks. This is considered as a baseline comprehension measurement. For this purpose, the data set contains examples that have already been labeled by the different networks. This means the results produced by the existing solution were used as inputs. For each network the balanced data set contained, respectively, 5,000 true positive, true negative, false positive, and false negative examples. As the n-gram length can play a role in the performances, their total count and distribution between valid and non-valid concepts in the test data set are reported in Table 4. The unbalanced distribution with respect to this aspect is clearly evident, but this is reflected in the dataset characteristics.

An evaluation of each network using the test data set described in the previous section should give a feeling on how well they behave, on top of the statistical evaluation. For this purpose, separate data sets were used to compute the labels given by the NN. Eight data sets (one for each NN configuration) were initialized, each having a precision and recall of 0.5 as output of the corresponding NN. Table 5 shows the general performance of concept recognition in the test data sets. Figure 5 lists the resulting precision, recall and $\mathrm{f} 1$ for different n-gram lengths in the validation sets. 
Table 4. Test data set distribution with respect to the n-gram length (Source [30]).

\begin{tabular}{l|l|l|l}
\hline Length & Total count & Concepts & Non concepts \\
\hline 1-gram & 90413 & $91.9 \%$ & $8.1 \%$ \\
\hline 2-gram & 164463 & $61.2 \%$ & $38.8 \%$ \\
\hline 3-gram & 107170 & $21.4 \%$ & $75.9 \%$ \\
\hline 4-gram & 52997 & $14.3 \%$ & $85.7 \%$ \\
\hline 5-gram & 20638 & $11.7 \%$ & $88.3 \%$ \\
\hline 6-gram & 8217 & $10.9 \%$ & $89.1 \%$ \\
\hline 7-gram & 3843 & $8.2 \%$ & $91.8 \%$ \\
\hline
\end{tabular}

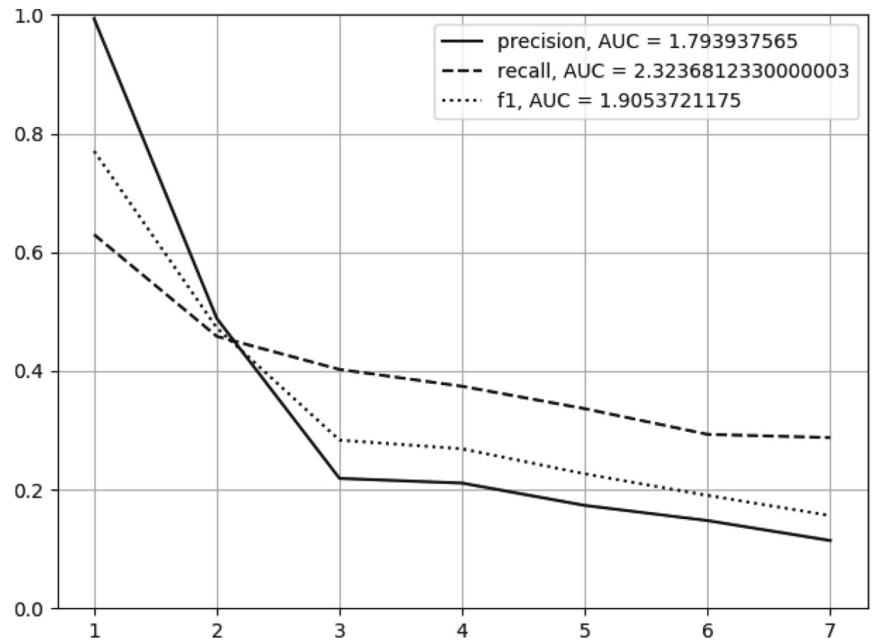

Fig. 5. Performances, with respect to n-gram length, of the CNN approaches, applied to a test dataset containing a list of 20,000 n-grams with an initial precision and recall output of the CNN of 0.5 for each CNN (reprinted from [30]).

In fact, the global performances of all networks negatively correlate with the n-gram length increases. Other than evaluating the whole test data set at once, the performance gaps of the different networks increased until some networks fell below 0.5. As before, V6H6, V6H3, and V0H3 outperformed their competitors; additionally, V3H0 performed almost equivalently. This suggests that they are more robust against unbalanced data and can achieve a more stable training process.

\section{Keyphrase Extraction Method}

With a CNN that recognizes concepts, it is possible to improve our keyphrase extraction (KPE) method. In this section, we will describe an initial statistical 
Table 5. Performance results after application to a list of n-grams that have been tagged by different CNNs and have been balanced to represent $25 \% \mathrm{TP}, \mathrm{FP}, \mathrm{TN}$ and FN examples (Source [30]).

\begin{tabular}{l|l|l|l}
\hline Network & Precision & Recall & F1 \\
\hline V6H6 & 0.650 & 0.323 & 0.432 \\
\hline V6H3 & 0.659 & 0.326 & 0.436 \\
\hline V3H0 & 0.731 & 0.317 & 0.442 \\
\hline V0H3 & 0.694 & 0.331 & 0.448 \\
\hline V6H0 & 0.702 & 0.334 & 0.452 \\
\hline V3H3 & 0.649 & 0.348 & 0.453 \\
\hline V6H1 & 0.668 & 0.353 & 0.462 \\
\hline V3H1 & 0.640 & 0.366 & 0.466 \\
\hline
\end{tabular}

approach for $\mathrm{KPE}$, the integration of $\mathrm{CNN}$ to it as a concept filter into the process, and we measure its performance impact.

\subsection{Keyphrase Extraction Based on Part of Speech Tagging}

We initially started with a purely POS-based procedure, where the Wikipedia corpus $^{1}$ is analyzed for frequent functional patterns, to determine valid keyphrases. Additionally, we inherited the term frequency $\mathrm{TF}()$ function and inverse document frequency $\operatorname{IDF}()$ from the information retrieval filed. They each respectively represent the frequency of appearance of a term in a document and the relative frequency of the term in the document with respect of the full corpus. The underlying assumption is that valid complex concepts normally share the same type of components in their names and that only some specific patterns are useful for keyphrase extraction. We then measured the ratio for each POS pattern (eg: (DT - JJ - NN - IN - NNP), instantiated by the great wall of China) between valid and invalid concept, such as in Eq. 7 and then weighted using TF-IDF to order them and extract the most significant ones, using the formula in Eq. 8.

$$
\begin{gathered}
\frac{P\left(\text { pattern } \mid C_{+}\right)}{P\left(\text { pattern } \mid C_{-}\right)} \\
\frac{D F(\boldsymbol{w})^{2}+\log \left(\frac{\# \text { docs }}{T F(\boldsymbol{w})+1}+1\right)}{\operatorname{size}(\text { doc })}
\end{gathered}
$$

The results demonstrated that this approach works very well with keywords (1-grams), but tend to eliminate keyphrases from the results. This is due two independent but concurrent phenomena: on one side, the part of speech filter removes many patterns including combinations as it tries to maximize the extraction precision; on the other side, the TF-IDF weight (in particular its Term

\footnotetext{
${ }^{1}$ https://dumps.wikimedia.org/enwiki/20181020.
} 
Frequency part) is highly biased for very frequent elements, and elementary form are generally more frequent than composed ones.

\subsection{Document-Based Keyphrase Extraction by Keywords Composition and Information-Based Weighting}

Our keyphrase extraction approach is inspired by a human oriented idea, observing the way a person would search for significant keyphrases in a text. Through a visual scan, an individual searches for familiar patterns (as sequences of keywords, maybe also skipping a finite number of intermediate elements). This is important as keyphrases are often arranged around important keywords or are a combination of important words, as can thus be seen in our keyphrase extraction example in Fig. 7. We apply this idea in our method by using TF-IDF-based keywords as anchors to generate keyphrase candidates. We also combined the previously described POS-based filtering into the keyphrase extraction pipeline. By analyzing statistical characteristics of the document analyzed, the function $P()$ measures the probability of appearance of an element inside it. It can be used to compute individual probabilities, such as $P\left(w_{1}\right)$, or to compute joint probability of two words, such as in the case of $P\left(w_{1}, w_{2}\right)$. The first function we used is the pointwise mutual information [16], which computes the amount of information two words shares:

$$
\operatorname{pmi}\left(w_{1}, w_{2}\right)=\frac{P\left(w_{1}, w_{2}\right)}{P\left(w_{1}\right) * P\left(w_{2}\right)}
$$

The limit of $p m i$ resides on the impossibility to use it for comparing single words values with sequences. To solve this issue, we adopted another metric, based on the concept of the information amount included in a single word, called self information [11]

$$
\operatorname{si}\left(w_{1}\right)=\frac{1}{P\left(w_{1}\right)}
$$

In order to compare the information gain for a words sequence $a$ and its extension $b$ (defined as $a$ with an additional consecutive word), we defined a function called average pointwise mutual information, which represents for the given sequence the average pointwise mutual information between each pair of consecutive words.

$$
\operatorname{avgP} M I(\boldsymbol{w})=\frac{1}{(n-1)} * \sum_{i=1}^{n-1} \operatorname{pmi}\left(w_{i}, w_{i+1}\right)
$$

Applying this functions allows us to answer the question: "How does the average information change if we add another word to the n-gram?", by comparing the avgPMI for $a$ and $b$. Additionally, we defined the mean probability of an n-gram, as the weighted sum of each word probability inside the words sequence:

$$
\bar{P}(\boldsymbol{w})=\frac{1}{n} \sum_{i=1}^{n} P\left(w_{i}\right)
$$


Furthermore to account for the positive co-occurrence of infrequent words, we defined the Inverse standard deviation of each word probability in an n-gram, which allows for higher weight to sequences which contains words with a similar probability:

$$
\operatorname{invsd}(\boldsymbol{w})=\frac{1}{\frac{1}{n} \sum_{i=1}^{n}\left|P\left(w_{i}\right)-\bar{P}(\boldsymbol{w})\right|}
$$

Eventually, the overall score combines the shown metrics into the following two formula for a generic sequence of words (including 1-grams (words) and ngrams):

$$
\operatorname{score}(\boldsymbol{w})= \begin{cases}\log (T F(\boldsymbol{w})) * \operatorname{si}(\boldsymbol{w}) & \boldsymbol{w} \text { is a proper word } \\ \log (T F(\boldsymbol{w})) *(\operatorname{avg} P M I(\boldsymbol{w})+\operatorname{invsd}(\boldsymbol{w})) & \boldsymbol{w} \text { is a proper n-gram }\end{cases}
$$

Given these functions, we defined the improved Keyphrase Extraction Method mode as follows. This process is illustrated in Fig. 6, where the pipeline from document to candidates tags is explained with reference to the following enumeration:

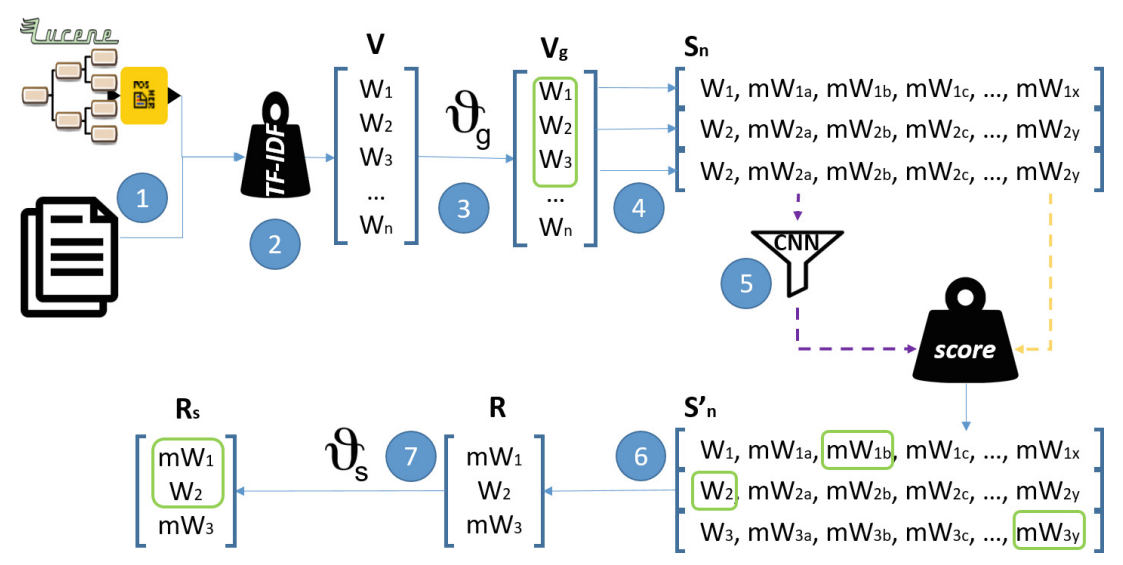

Fig. 6. The process flow of our proposed approach: 1-Grams are represented by W, and multigrams are represented by $\mathrm{mW}$. The alternative paths with and without CNN usage are depicted as dashed arrows, respectively in black and yellow. Green highlighting is used to show the selected element in each step. (Color figure online)

1. We developed a 1-gram index on Wikipedia corpus and filtered it by part of speech as described in Subsect. 4.1 to create a keyword reference model, indicated as $R$.

2. Then, the approach found the most important words, in their singular form, based on their TF-IDF measure, and considered the elements in this ordered set $V$ as keywords (1-grams). 
3. A generation threshold $\theta_{g}$ limited the number of keywords to guarantee that a certain percentage of the information was conserved. For example $\theta_{g}=0.8$ preserves around $80 \%$ of the information. This restricted set is called $V_{g}$.

4. On the original text we computed $S$ as the set of all n-grams composed by maximum 7 elements contained in the respective document. For each element $W$ in $V_{g}$ we collected from $S$ the candidates combinations containing $W$ that were delimited by valid keywords. For example, given $W=$ States the combination President of the United States of America is a valid candidate, under the assumption $\{$ President, America $\} \subseteq S$. Note that gaps are also allowed in valid combinations to allow inclusion of other POS elements such as prepositions, conjunctions, adverbs, and pronouns. This new multi set was named $S_{n}$.

5. The optional CNN concept recognition phase was applied at this level to improve the extracted keywords by filtering them for having a high probability of being concepts. In the following section, the quality of keyphrase extraction was evaluated with and without CNN-based concept recognition filtering.

6. A weighting procedure was adopted following the formula in Eq. 14. This allowed us to rank each candidate combination with respect to each element $W$. The one with the highest weight was then selected as representative. The resulting set was designated as $S_{n}^{\prime}$

7. On the set $R$ produced by the previous step, a dual filtering procedure was applied as the final stage based on the $\theta_{s}$ parameter: this criterion was applied on the original $V_{g}$, giving the best candidate for each initial keyword until the coverage of the expected information percentage. This allowed us to restrict

\section{Database administrator_C.txt}

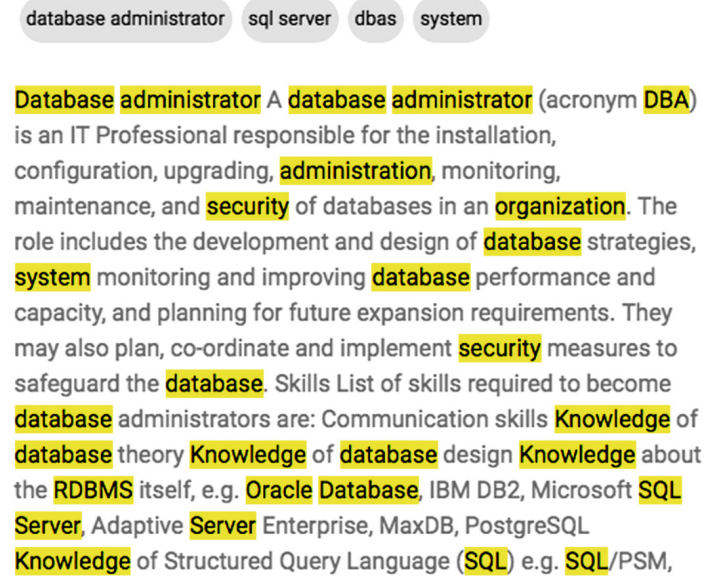

Fig. 7. Keyphrase extraction result for the Wikipedia entry "Database Administrator", as a screenshot in the UI of a prototypical software implementation. 
the final set of suggested keyphrases, called $R_{s}$, in order to reduce to a set with higher information ratio.

As a first visual example, Fig. 7 shows the resulting keyphrases extracted for the Wikipedia article Database Administrator. In the text, the TF-IDFbased single keywords are highlighted. The goal of our method was to recognize frequent and important combinations of keywords and use them for composing n-gram keyphrases.

In a more detailed example, we will take a paragraph from a news website and present the proposed process using it. The website chosen is "the Washington Post" with an article titled "The government is rolling out 2-factor authentication for federal agency dot-gov domains" 2 . It is useful to note that this is not part of our corpus guaranteeing by construction the prevention of over-fitting effects. The selected paragraph is as follows:

Federal and state employees responsible for running government websites will soon have to use two-factor authentication to access their administrator accounts, adding a layer of security to prevent intruders from taking over dot-gov domains.

Table 6. After step 2, extracted keywords are ordered by descending importance, together with their cumulative TF-IDF weight. This is the $V$ vector. In step 3 , using $\theta_{g}=0.84$, the vector is limited to the top keywords with $\sum($ weight $)<\theta_{g}$, named $V_{g}$.

\begin{tabular}{l|l|l|l|l|l}
\hline Keyword & weight & $\sum($ weight $)$ & Keyword & weight & $\sum$ (weight $)$ \\
\hline federal & 0.130 & 0.130 & employee & 0.048 & 0.746 \\
\hline dot-gov & 0.130 & 0.260 & account & 0.043 & 0.789 \\
\hline two-factor & 0.116 & 0.376 & security & 0.043 & 0.832 \\
\hline authentication & 0.087 & 0.464 & responsible & 0.042 & 0.874 \\
\hline intruder & 0.076 & 0.540 & access & 0.042 & 0.916 \\
\hline administrator & 0.055 & 0.595 & website & 0.041 & 0.957 \\
\hline domain & 0.054 & 0.648 & government & 0.025 & 0.982 \\
\hline layer & 0.050 & 0.698 & state & 0.018 & 1.000 \\
\hline
\end{tabular}

As result of step 2, the set of limited extracted keywords $V_{g}$ is presented in Table 6 together with their TF-IDF weight, rescaled to the unitary total, and its cumulated sum. In Step 3, the generation threshold $\theta_{g}$ is used to enforce the consideration of keywords from $V$ till the cumulated TF-IDF measure do not exceed the parameter settled. Based on the limited set of keywords $\left(V_{g}\right)$ the following potential concepts are found inside the text. N-grams with a length between 1 and 7 are allowed. Each valid concept has to start and end with one element of the limited set of keywords $V_{g}$, but inside the n-gram any word existing in the original text is allowed to appear. The result is shown in Table 7.

\footnotetext{
${ }^{2}$ The permanent link for the selected news item is https://perma.cc/PF53-SY2L.
} 
Table 7. Step 4: Extracted candidate concepts for each keyword. This is indicated as $S_{n}$. The 1-grams corresponding to the keywords (first column) are not repeated.

\begin{tabular}{||c||c|c|}
\hline Keyword & candidate Concept & candidate Concept \\
\hline \hline federal & federal and state employee & \\
dot-gov & dot-gov domain & \\
two-factor & two-factor authentication & \\
authentication & two-factor authentication & \\
intruder & security to prevent intruder & layer of security to prevent intruder \\
administrator & administrator account & \\
domain & dot-gov domain & \\
layer & layer of security & layer of security to prevent intruder \\
employee & federal and state employee & \\
account & administrator account & \\
security & layer of security & layer of security to prevent intruder \\
\hline
\end{tabular}

Step 5 is an optional part of the pipeline that preserves only the valid concepts by applying CNN filtering on the extracted candidates. In our example, using the best CNN configuration, this step eliminates the candidate concepts "federal and state employee", "security to prevent intruder", and "layer of security to prevent intruder" because they have a low probability of being a concept. From this point on, every candidate (also the 1-grams, previously known as keywords) are called concept; we proceeded with two cases, namely the result obtained with and without CNN filtering.

On step 6, the scoring of these candidates is performed following the formula in Eq. 14. This produces an order amongst the candidates for each keyword and then the highest scored concept is selected. Table 8 presents the selection of the set of concepts to use in vector $R$ : grayed lines indicates elements filtered out by the CNN, as improbable concepts. The suit symbols indicates the selection of a specific concept to represent a keyword (in the columns): spade (\$) is used when the selection is common to both cases (with and without CNN filtering out), club (\$) pinpoints the top candidate without CNN filtering out, and heart $(\nabla)$ is the highest remaining candidate whenever the previous concepts are removed by $\mathrm{CNN}$ filtering. Eventually, diamonds $(\diamond)$ point to candidates that are not selected by either method.

Eventually, in step 7 the selection threshold is applied, to project back to the original cumulative information comparison. In our example, we use $\theta_{s}$ set to 0.72 , that means in Table 6 to consider the top 8 elements (equivalent to the first column, till "layer", where the cumulated information is 0.698), that translates in Table 8 into the first 8 columns. This is the final result $R_{s}$, that is represented, by comparison, into Table 9 . 
Table 8. Step 6: The selection of the elements to compose the result $R$, based on the scoring function. Combinations marked by are selected in both cases (with and without CNN filtering); $\$$ represents the top candidates without using CNN; $\odot$ indicates the highest remaining candidate after the CNN filtering step; $\diamond$ represents candidates that are not selected by either method. The vertical separation refers to step 7 , where a selection threshold $\theta_{s}=0.74$ is applied, leaving only the first 8 columns in the resulting final set, know as $R_{s}$.

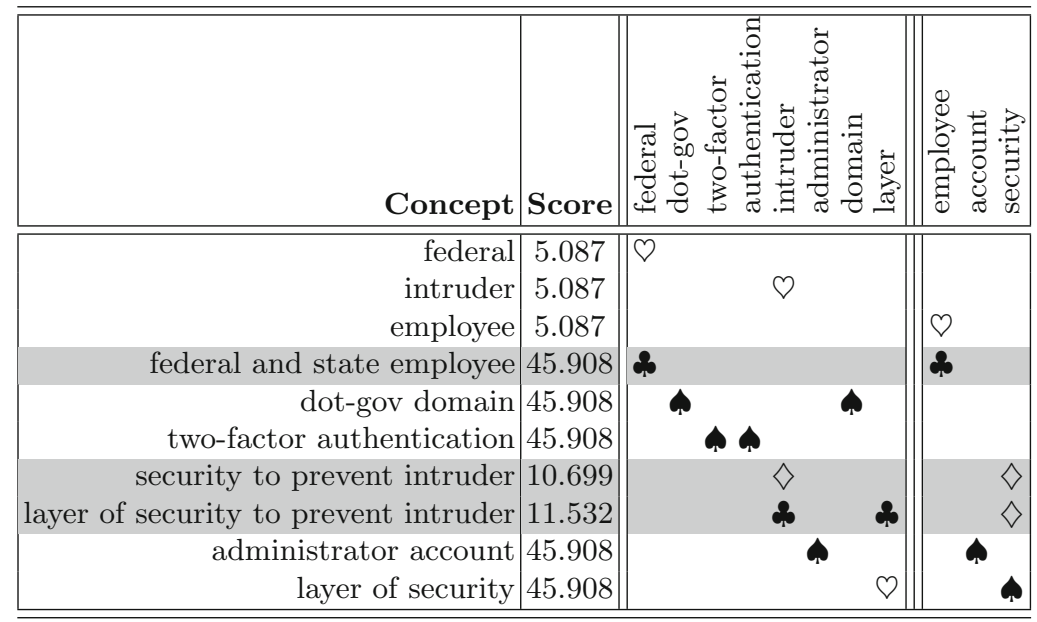

Table 9. The final candidate concepts for our small example comparing the case without (left) and with CNN (right). The number of candidates is not the same, as one concept can be associated to more than one keywords such as in the case of "layer of security to prevent intruder" in the removed concepts, here.

\begin{tabular}{l|l}
\hline Concepts & $\begin{array}{l}\text { With CNN (best } \\
\text { configuration) }\end{array}$ \\
\hline Without CNN & federal \\
federal and state employee & dot-gov domain \\
dot-gov domain & two-factor authentication \\
two-factor authentication & intruder \\
layer of security to prevent & administrator account \\
intruder & layer of security \\
administrator account &
\end{tabular}




\subsection{Performance Impact of Adding CNN-Filter to Keyphrase Extraction}

We can test the performance improvement by measuring the difference in precision, whenever we enhance the statistical keyphrase extraction with CNN-based concept recognition. If the $\mathrm{CNN}$ is applied as a filter, the n-gram keyphrase candidates can be improved in quality if the CNN can accurately predict concept-hood for n-grams. To test this, we implemented the following experimental setup. As a test set, we use 132.737 English Wikipedia articles of good quality: $18.487 \mathrm{FA}$ (features articles), 27.738 GA (good articles) and 86.512 of quality level A. For every article, the keywords were extracted according to the process shown in Fig. 6 using different parameter configurations:
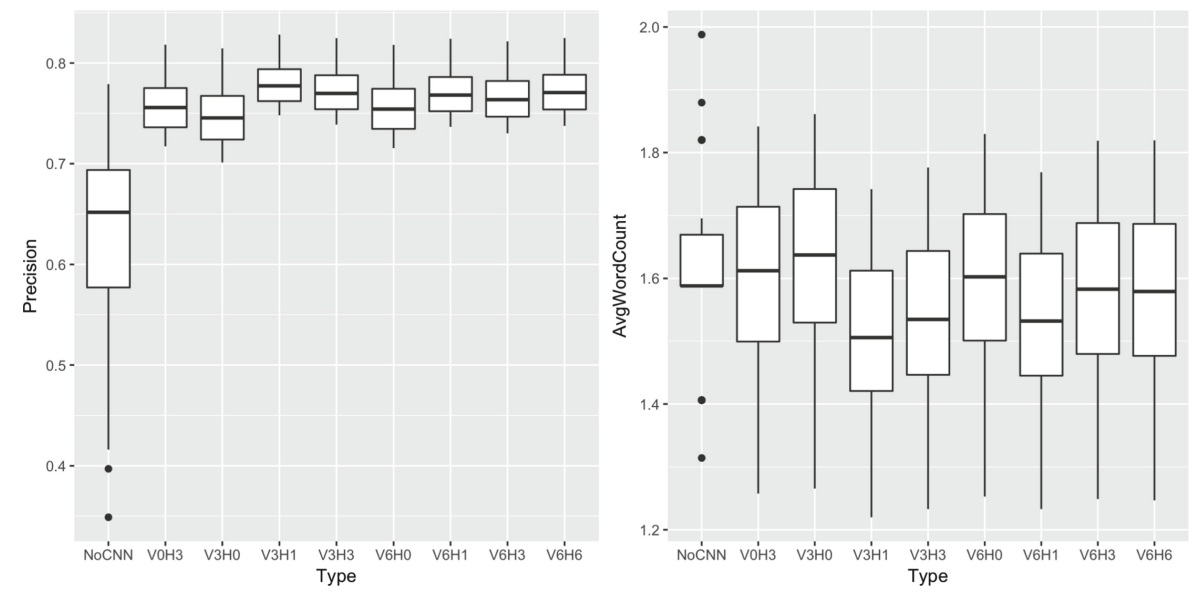

Fig. 8. Whisker-Boxplots of average precision and average keyphrase length (AvgWordCount) for every CNN configuration as discussed in Subsects. 3.3 and 3.4, compared to a configuration without CNN (the most left element, labeled as NoCNN).

- 10 levels of selection threshold $\theta_{g}: 0.48-0.84$ in discrete steps of 0.04 )

- 10 levels of generation threshold $\theta_{s}$ : again, 0.48-0.84 in discrete steps of 0.04, with the restriction of $\theta_{s} \leq \theta_{g}$

- 9 Types of keyphrase extraction, one without CNN and 8 with different CNN configurations, as described in Sect. 3.

For every Wikipedia article and for every parameter configuration $\left(\theta_{s}, \theta_{g}\right.$, Type), we collected the results as a list of extracted keyphrases. Now, for quality assessment, this list was compared to the list of concepts that have a Wikipedia entry. We assumed for our test that qualitatively good n-gram keyphrases are those that represent concepts, and if a keyphrase has a corresponding Wikipedia entry, we are certain that it represents a concept. Accordingly, a true positive (TP) is an extracted keyphrase that has a Wikipedia entry, and a false positive (FP) is a keyphrase without one. The precision of concept recognition in the list of extracted keyphrases for a document is then computed as the number of 
true positives divided by the number of keyphrases, meaning $\frac{\# T P}{(\# T P+\# F P)}$. We then summed up the document-level precisions to an average precision (macroaverage) grouped by parameter combination. This allows us to compare the quality performance of different configurations. In the following paragraphs, we present the resulting data of these experiments.

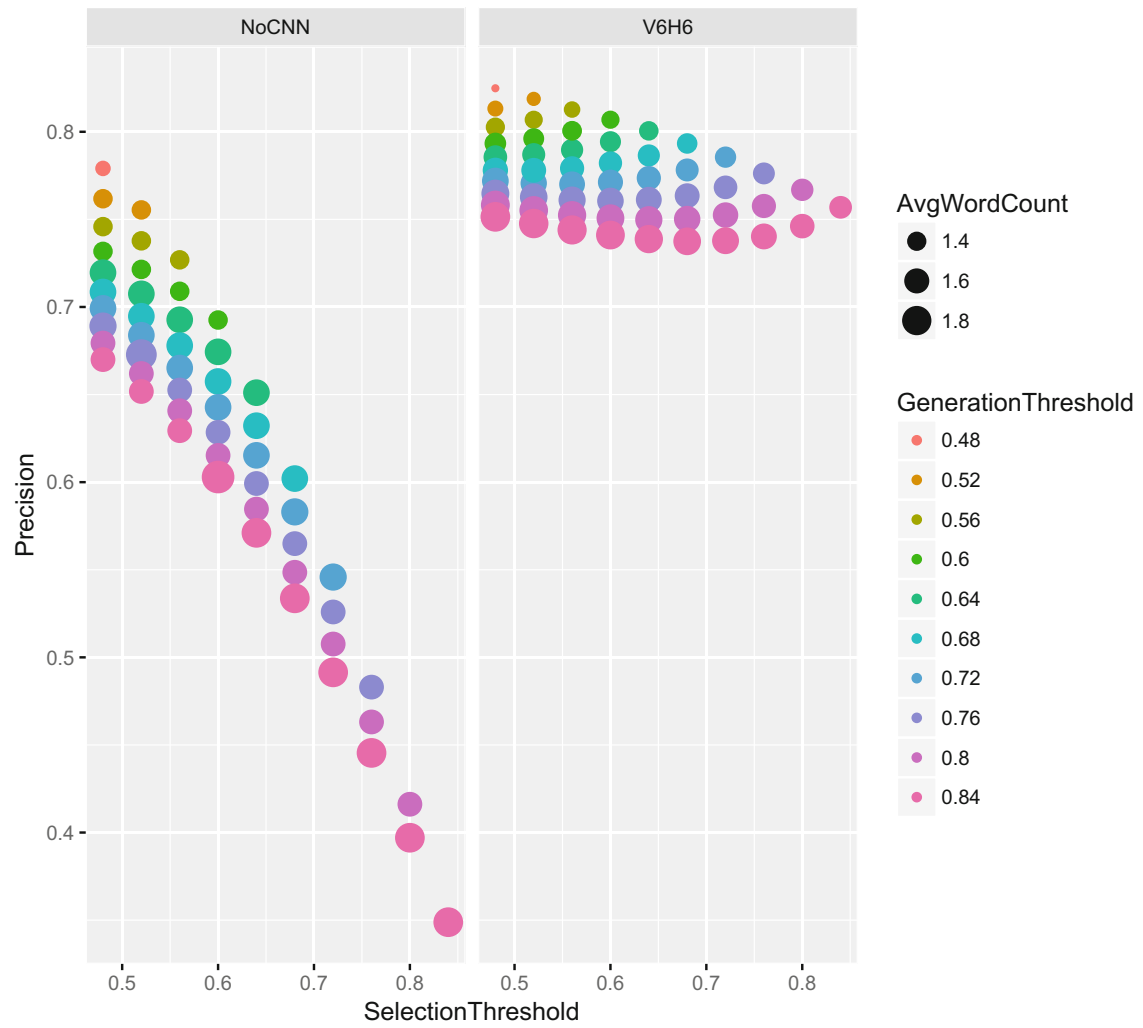

Fig. 9. Resulting precision and average keyphrase length (labeled AvgWordCount) without CNN (left) and with the optimal CNN configuration V3H1 (right), for different values of $\theta_{g}$ (color) and $\theta_{s}$ (independent axes). (Color figure online)

The left side of Fig. 8 shows that all CNN provide a significant improvement of concept precision compared to keyphrase extraction without CNN, and in particular the types V3H1, V3H3 and V6H6 showed the best improvements. Additionally, there are no more outliers (in particular towards the low end of the distribution) that is also a very good improvement. On the right side of Fig. 8 the average length $(n)$ of the generated $n$-gram keyphrases is compared. As we can observe, some configurations of CNN tends to privilege shorter word composition as candidate keyphrases (such as V3H1, V3H3 and V6H1) whether others improves also on this aspect. In our use case of automatic tagging, precision is the most important criterion, in the sense of quality rather than quantity. 

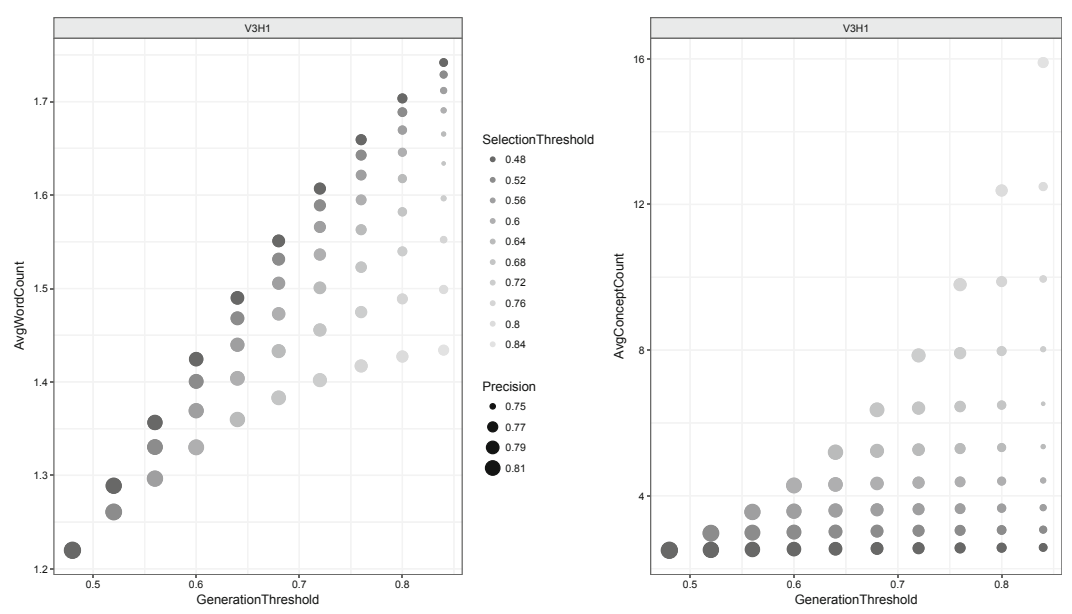

Fig. 10. Average number of words per keyphrase (AvgWordCount), average number of keyphrases per document (AvgConceptCount), and resulting precision (radius) of the optimal CNN configuration V3H1 for different values of $\theta_{g}$ (independent axes) and $\theta_{s}$ (grey scale).

Therefore, V3H1, the configuration that increases the precision the most, is considered as the optimal representative of CNN in our analysis, from this point on. We can now analyze the performance of our solution with respect to the different parameters used to tune it. The left side of Fig. 9 represents the concept precision achieved without CNN for different values of selection threshold $\theta_{s}$ that controls the amount of information preserved at the end of the pipeline, by selecting the top slice of keyphrase candidates. The right side of Fig. 9 compares this precision grouped by $\theta_{s}$ for the optimal CNN configuration V3H1. It is immediately evident that the base case (without $\mathrm{CNN}$ ) is really sensible to increases in $\theta_{s}$ value because the precision drops as soon as you enlarge the pool as false positives more frequently appear in the candidate lists. On the contrary, the CNN seems to be largely immune from this effect, and it even appears to improve the precisions for larger values of $\theta_{s}$. On the same graph, encoded by the point radius is presented the average length of generated keyphrase that show a very limited decrease of it, due to the introduction of the CNN in the pipeline. The color code represents the generation threshold $\theta_{g}$, and what can be observed is that with a higher value of $\theta_{g}$, the precision increases but also the average $n$-gram length (AvgWordCount) decreases.

In Fig. 10 the effects of the generation threshold $\theta_{g}$ on the length (left) and number (right) of candidates keyphrases is illustrated. This analysis is performed only for the pipeline including the CNN, in configuration V3H1. The color encodes the value of the selection threshold $\theta_{s}$, whether the point size maps the achieved precision. An increase in $\theta_{g}$ produces, as expected, longer keyphrases, as more composed sequences emerges from the candidates; at the same time, the number of produced keyphrases positively correlate with the 
Table 10. Result Comparison with and without CNN of the integrated pipeline with the same configuration. Notable is the increase in precision measure.

\begin{tabular}{l|l|l|l|l|l}
\hline$\theta_{s}$ & $\theta_{g}$ & Type & Precision & AvgWordCount & AvgConceptCount \\
\hline 0.64 & 0.64 & NoCNN & 0.651 & 1.669 & 4.932 \\
\hline 0.64 & 0.64 & V3H1 & 0.806 & 1.360 & 5.190 \\
\hline
\end{tabular}

selection threshold $\theta_{s}$, as this causes more base keywords to be maintained, and they can be composed to define $n$-gram concepts. Also, the higher the selection threshold, the lower the average length of the extracted keyphrases.

If we assume an optimal number of five extracted keyphrases on average per document, we have an optimal configuration with parameters $\theta_{s}=\theta_{g}=0.64$. As shown in Table 10, using this configuration we get a concept precision of 0.65 without CNN compared to a concept precision of 0.8 with a CNN with 3 horizontal and 1 vertical convolution layers, which is a clear improvement.

As an external evaluation, we measured precision and recall on the keyphrase extraction gold standard data sets Inspec and DUC according to the method described by Eiholzer [6]. First we tested for the optimal configuration. As shown on the right side of Fig. 11, the optimal CNN type of V3H1, consistent to the internal evaluation shown in Fig. 8. The left side shows an optimal $\theta_{g}$ parameter at 0.48 consistent to the internal evaluation shown on the right side of Fig. 9 . Second, we compared our approach against data published by Bennani et al. [3]. Our approach with optimal configuration shows the highest precision (Table 11).
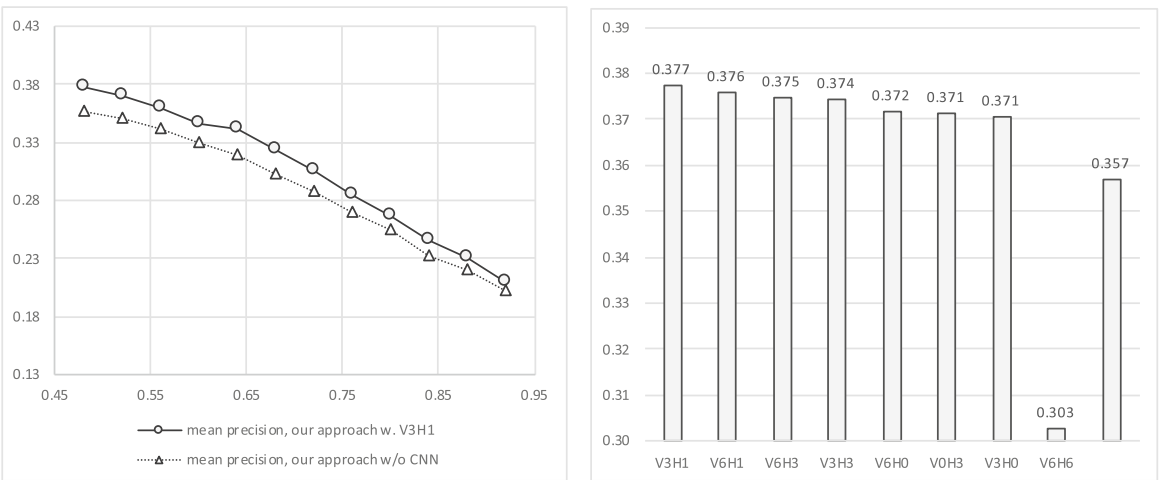

Fig. 11. The optimal configuration (left: $\mathrm{X}=\theta_{g}$, right: $\mathrm{X}=\mathrm{CNN}$ type) according to the mean precision in an external evaluation of our approach on Inspec and DUC keyphrase extraction gold standard is $\theta_{g}=0.48$ and $\mathrm{CNN}$ type $=\mathrm{V} 3 \mathrm{H} 1$. 
Table 11. Benchmarking of our approach $\left(\theta_{g}=0.48\right)$ on DUC and Inspec, on the Precision, Recall and F1-measure for top 5 keyphrase extraction. Approach ${ }_{A}$ is without CNN and $\mathrm{Approach}_{B}$ is with CNN in configuration V3H1.

\begin{tabular}{l|l|l|l|l|l|l|l|l|l}
\hline \multirow{2}{*}{ Algorithm } & \multicolumn{4}{l}{ Inspec } & \multicolumn{4}{l|}{ DUC } & \multicolumn{3}{l}{ Mean } \\
\cline { 2 - 10 } & Precis. & Recall & F1 & Precis & Recall & F1 & Precis. & Recall & F1 \\
\hline TextRank & 0.249 & 0.105 & 0.147 & 0.198 & 0.123 & 0.152 & 0.224 & 0.114 & 0.150 \\
\hline SingleRank & .382 & 0.233 & 0.289 & 0.303 & 0.195 & 0.237 & 0.343 & 0.214 & 0.263 \\
\hline TopicRank & 0.333 & 0.199 & 0.249 & 0.278 & 0.183 & 0.221 & 0.306 & 0.191 & 0.235 \\
\hline Multipartite & 0.346 & 0.205 & 0.258 & 0.295 & 0.194 & 0.234 & 0.321 & 0.200 & 0.246 \\
\hline EmbedRank d2v & 0.415 & $\mathbf{0 . 2 5 4}$ & $\mathbf{0 . 3 1 5}$ & 0.309 & $\mathbf{0 . 1 9 7}$ & $\mathbf{0 . 2 4 0}$ & 0.361 & $\mathbf{0 . 2 2 5}$ & $\mathbf{0 . 2 7 7}$ \\
\hline Approach $_{A}$ & 0.412 & 0.145 & 0.214 & 0.301 & 0.170 & 0.217 & 0.357 & 0.157 & 0.216 \\
\hline Approach $_{B}$ & $\mathbf{0 . 4 4 2}$ & 0.172 & 0.248 & $\mathbf{0 . 3 1 3}$ & 0.172 & 0.227 & $\mathbf{0 . 3 7 7}$ & 0.175 & 0.238 \\
\hline
\end{tabular}

\section{Conclusion}

\subsection{Insights and Lessons Learned}

Our research contributes a soft computing method to recognize word sequences as concepts to filter the candidate concepts for key-phrase extraction. We trained a convolutional neural network using arrays of Word2Vec based embedding vectors (n-gram-matrices) as analytic signals and the existence of a Wikipedia title equal to the n-gram as a binary training signal to teach the CNN if an n-gram is a concept or not.

For the application of concept recognition to automatic tagging of documents, we are interested in high concept precision because false positives, that are keyphrases that are not concepts, decrease user acceptance of such a system. Also we are interested in $n$-gram concepts (with $n>1$ ), as significant keyphrases usually consist of more than one word. Our experiments show that the concept recognition precision decreased with higher number of words in the n-grams, as from Fig. 5 .

We contribute a statistical method for keyphrase extraction, and a solution to integrate $\mathrm{CNN}$-based concept recognition to this keyphrase extraction to increase concept precision, the percentage of extracted keyphrases that are concepts. The experiments described in Subsect. 4.3 demonstrate that statistical keyphrase identification can be consistently improved using CNN-based concept recognition. We demonstrated an increase of concept precision for the extracted keyphrases calculated for Wikipedia articles, if extraction pipeline is combined with a CNN-based concept recognition filter. The data in Fig. 8 demonstrates that the percentage of automatically extracted keyphrases that are actual concepts was significantly enhanced by application of the CNN. Furthermore as seen in Fig. 9, whereas without CNN the average precision dropped quickly with increasing the number of keyphrases per document, this precision was more stable even with more extracted keyphrases adopting CNN filtering. Furthermore, 
as seen in Fig. 10, in a bench-marking experiment based on two key phrase extraction gold standard data sets, our approach with CNN-based concept recognition showed the highest precision compared to other methods found in the literature, even though it does not yield the highest recall. Since for automatic tagging, precision is more important than recall, because quality matters more than quantity, this result is optimal for our use case.

\subsection{Outlook}

There are several aspects to be considered for further research projects. Firstly, stemming from the gold standard could lead to a more general evaluation. Experimenting with different word features could increase the performance significantly. Secondly, we are currently experimenting with generating humanunderstandable features for words and n-grams based on co-occurrence statistics and explicit semantic analysis to generate a different type of word embedding. Thirdly we are comparing NN to Bayesian models and other types of models for concept recognition training towards getting a better understanding of the classification process. Fourthly instead of using a balanced list of concepts and non-concepts, the training data will be generated by going through the text corpus word by word. Thus, the network would be trained with n-grams in the sequence they appear in the text. Thus, frequent n-grams would be getting more weight. And last but not least, changing the input consideration and using a recurrent neural network instead of a CNN could improve the results.

\section{Note}

This is an extended version of a conference paper [30]. Equations (1), (2), (3), (4), (5) and (6) are reprinted with authors' permission from it.

Acknowledgements. This research has been funded in part by the Swiss Commission for Technology and Innovation (CTI) as part of the research project Feasibility Study X-MAS: Cross-Platform Mediation, Association and Search Engine, CTI-No. 26335.1 PFES-ES. We thank Benjamin Haymond for proof-reading and copy-editing of our work.

\section{References}

1. Beliga, S., Metrovic, A., Martinic-Ipsic, S.: An overview of graph-based keyword extraction methods and approaches. J. Inf. Organ. Sci. 39, 1-20 (2015)

2. Bengio, Y.: Practical recommendations for gradient-based training of deep architectures. CoRR abs/1206.5533 (2012). http://arxiv.org/abs/1206.5533

3. Bennani-Smires, K., Musat, C., Jaggi, M., Hossmann, A., Baeriswyl, M.: EmbedRank: unsupervised keyphrase extraction using sentence embeddings. CoRR abs/1801.04470 (2018). http://arxiv.org/abs/1801.04470

4. Dalvi, N., et al.: A web of concepts. In: Proceedings of the Twenty-Eighth ACM SIGMOD-SIGACT-SIGART Symposium on Principles of Database Systems, PODS 2009, pp. 1-12. ACM, New York (2009). https://doi.org/10.1145/1559795. 1559797 
5. Das, B., Pal, S., Mondal, S.K., Dalui, D., Shome, S.K.: Automatic keyword extraction from any text document using n-gram rigid collocation. Int. J. Soft Comput. Eng. (IJSCE) 3(2), 238-242 (2013)

6. Eiholzer, M.: Method engineering for automatic tagging with inductive fuzzy classification. Master's thesis, School of Computer Science, Lucerne University of Applied Sciences and Arts, Rotkreuz, Switzerland (2019)

7. Fürnkranz, J.: A study using n-gram features for text categorization. Austrian Res. Inst. Artif. Intell. 3(1998), 1-10 (1998)

8. Google: Googlenews-vectors-negative300.bin.gz (2013). https://drive.google.com/ file/d/0B7XkCwpI5KDYNINUTTISS21pQmM/edit. Accessed 15 Jan 2018

9. Hughes, M., Li, I., Kotoulas, S., Suzumura, T.: Medical text classification using convolutional neural networks. arXiv preprint arXiv:1704.06841 (2017)

10. Hulth, A.: Improved automatic keyword extraction given more linguistic knowledge. In: Proceedings of the 2003 Conference on Empirical Methods in Natural Language Processing (2003)

11. Jagarlamudi, J., Pingali, P., Varma, V.: Query independent sentence scoring approach to DUC 2006. In: Proceeding of Document Understanding Conference (DUC) (2006)

12. Joulin, A., Grave, E., Bojanowski, P., Mikolov, T.: Bag of tricks for efficient text classification. arXiv preprint arXiv:1607.01759 (2016)

13. Kalchbrenner, N., Grefenstette, E., Blunsom, P.: A convolutional neural network for modelling sentences. CoRR abs/1404.2188 (2014). http://arxiv.org/abs/1404. 2188

14. Kim, Y.: Convolutional neural networks for sentence classification. arXiv preprint arXiv:1408.5882 (2014)

15. Lee, S., Kim, H.: News keyword extraction for topic tracking. In: 2008 Fourth International Conference on Networked Computing and Advanced Information Management, vol. 2, pp. 554-559, September 2008. https://doi.org/10.1109/NCM. 2008.199

16. Liu, Y., Shi, M., Li, C.: Domain ontology concept extraction method based on text. In: 2016 IEEE/ACIS 15th International Conference on Computer and Information Science (ICIS), pp. 1-5. IEEE (2016)

17. Liu, Z., Li, P., Zheng, Y., Sun, M.: Clustering to find exemplar terms for keyphrase extraction. In: Proceedings of the 2009 Conference on Empirical Methods in Natural Language Processing: Volume 1. Association for Computational Linguistics (2009)

18. Lopez, M.M., Kalita, J.: Deep learning applied to NLP. CoRR abs/1703.03091 (2017). http://arxiv.org/abs/1703.03091

19. Mihalcea, R., Tarau, P.: TextRank: bringing order into texts. In: Proceedings of the 2004 Conference on Empirical Methods in Natural Language Processing, EMNLP $2004(2004)$

20. Mikolov, T., Chen, K., Corrado, G., Dean, J.: Efficient estimation of word representations in vector space. arXiv preprint arXiv:1301.3781 (2013)

21. Nair, V., Hinton, G.E.: Rectified linear units improve restricted Boltzmann machines. In: Proceedings of the 27th International Conference on Machine Learning (ICML 2010), pp. 807-814 (2010)

22. Pang, L., Lan, Y., Guo, J., Xu, J., Wan, S., Cheng, X.: Text matching as image recognition. In: AAAI, pp. 2793-2799 (2016)

23. Parameswaran, A., Garcia-Molina, H., Rajaraman, A.: Towards the web of concepts: extracting concepts from large datasets. Proc. VLDB Endow. 3(1-2), 566$577(2010)$ 
24. Rong, X.: word2vec parameter learning explained. arXiv preprint arXiv:1411.2738 (2014)

25. Rose, S., Engel, D., Cramer, N., Cowley, W.: Automatic keyword extraction from individual documents. In: Berry, M.W., Kogan, J. (eds.) Text Mining: Applications and Theory. Wiley, Hoboken (2010)

26. Siegfried, P., Waldis, A.: Automatische generierung plattformübergreifender wissensnetzwerken mit metadaten und volltextindexierung, July 2017. http://www. enterpriselab.ch/webabstracts/projekte/diplomarbeiten/2017/Siegfried.Waldis. 2017.bda.html

27. Simonyan, K., Zisserman, A.: Very deep convolutional networks for large-scale image recognition. arXiv preprint arXiv:1409.1556 (2014)

28. Song, Y., et al.: Real-time automatic tag recommendation. In: Proceedings of the 31st Annual International ACM SIGIR Conference on Research and Development in Information Retrieval, pp. 515-522. ACM (2008)

29. Srivastava, N., Hinton, G., Krizhevsky, A., Sutskever, I., Salakhutdinov, R.: Dropout: a simple way to prevent neural networks from overfitting. J. Mach. Learn. Res. 15(1), 1929-1958 (2014)

30. Waldis, A., Mazzola, L., Kaufmann, M.: Concept extraction with convolutional neural networks. In: Proceedings of the 7th International Conference on Data Science, Technology and Applications - Volume 1: DATA, pp. 118-129. INSTICC, SciTePress (2018). https://doi.org/10.5220/0006901201180129

31. Westphal, C., Pei, G.: Scalable routing via greedy embedding. In: INFOCOM 2009, pp. 2826-2830. IEEE (2009)

32. Zhang, Q., Wang, Y., Gong, Y., Huang, X.: Keyphrase extraction using deep recurrent neural networks on Twitter. In: EMNLP (2016) 